\title{
Supplementation of Optimum Nutrient-Dense Formula to Modulate HIV Infection in Resource Limited Settings
}

\author{
Ihab Tewfik ${ }^{1}$, Abraham Mainaji Amlogu ${ }^{* 1,2}$, Sundus Tewfik ${ }^{3}$ and Charles Wambebe \\ ${ }^{1}$ Food, Nutrition and Public Health Division, University of Westminster, UK \\ ${ }^{2}$ State House Medical Center, Nigeria \\ ${ }^{3}$ The International Forum for Public Health, UK \\ ${ }^{4}$ Department of Pharmaceutical Sciences, Tswane University of Technology, South Africa
}

*Corresponding author: Abraham Mainaji Amlogu, Food, Nutrition and Public Health Division, School of Life Sciences, University of Westminster, London, 115 New Cavendish Street, W1W 6UW, UK.

To Cite This Article: Abraham Mainaji Amlogu. Supplementation of Optimum Nutrient-Dense Formula to Modulate HIV Infection in Resource Limited Settings. Am J Biomed Sci \& Res. 2019 - 5(1). AJBSR.MS.ID.000878. DOI: 10.34297/AJBSR.2019.05.000878

Received: 堽August 27, 2019; Published: 制 September 09, 2019

\begin{abstract}
HIV infection attenuates food intake and increases energy requirements through increases in resting energy expenditure. Equally, it triggers nutrient mal-absorption, negative nitrogen balance and metabolic alterations that lead to weight loss and wasting. Substantiating evidences from the literature highlighted the role of nutrient-dense meals in enhancing nutrition status of people living with HIV (PLHIV) and improving some HIVrelated outcomes. Despite limited resources, West African countries are renowned with various micro and macronutrients in commonly available food sources which contain antioxidants and relevant essential vitamins and minerals. Such food sources need to be appropriately analyzed vis-a-vis their potentials for use in the management of HIV/AIDS. Hence, nutrition intervention programs that employed nutrient-dense meals -NDMs [e.g. Amtewa meal] were effectively integrated in the management of PLHIV who are on highly active antiretroviral therapy (HAART). Prolonged use of NDMs [over 12 weeks] has demonstrated to attenuate and possibly obviate the progression to AIDS.
\end{abstract}

\section{Rationale and Background}

Despite the effectiveness of highly active antiretroviral therapy (HAART), there are growing evidence that HIV-related wasting remains an important co-morbidity factor among many patients in resource limited settings [1,2]. Prior analyses have examined the impact of malnutrition (BMI $18.5 \mathrm{~kg} / \mathrm{m} 2$ ) on the response to antiretroviral therapy [ART] in resource limited settings [3,4]. ARTs have been shown to reverse under-nutrition in HIV/AIDS but are usually used at the later stages of the disease when the patients are moribund [5,6]. For instance, $75 \%$ of Nigerians infected with HIV do not require ART, but nutritional assistance to maintain the immune system, sustain healthy levels of physical activity and for optimal quality of life. The implication of the reality on ground is that all the interventions now are grossly unable to cope with the treatment of those who require ARTs urgently. Hence, it is therefore important to articulate appropriate nutrition intervention programmes which could be integrated in the management of people living with HIV who are presently on HAART, with the aim of attenuating and possibly obviating the progression to AIDS.

\section{Current Recommendation and Intervention Programmes}

The 2019 epidemic update revealed slow but steady progress on averting HIV-related deaths, disease progression, and new HIV infections, progress that resulted from expanded access to antiretroviral therapy, antiretroviral prophylaxis, and safe infantfeeding interventions [7]. Of all people living with HIV, 79\% [6792\%] knew their status, 62\% [47-74\%] were accessing treatment and 53\% [43-63\%] were virally suppressed [8]. However, HIV intervention programmes at international level have some limitations. These include the following:

1. Only limited data exist about the prevalence of metabolic complications on long term use of ART in resource-limited settings and how best to manage them clinically; the impact of nutritional status upon these complications especially in those that are chronically undernourished needs to be established. 
2. Evaluation of the impact of acute or chronic severe malnutrition in children on CD4 levels, response to ART or likelihood of ART side effects.

3. Data are needed about the potential effect of ART on the nutritional needs of lactating women particularly in resource limited settings where breastfeeding is the predominant mode of infant feeding.

4. Evaluation of the potential impact of ART on growth and nutritional status of infected and uninfected infants born of HIV infected mothers in areas where malnutrition is common is a high priority.

5. Data are needed on the interaction between poor nutritional status of bone related nutrients (calcium and vitamin D) and ART in resource limited settings.

6. Because of the potential long-term health consequences of the metabolic complications of ART, appropriate research is needed on potential management for lipodystrophy and related conditions (e.g. diet or lifestyle changes) in adults and children, particularly those in resource limited settings.

The revised recommendation for ART included an earlier start to treatment for all HIV-infected individuals [e.g. test-and-treat strategy] and those with active tuberculosis, or active chronic hepatitis B [9]. They are based on evidences of both individual and public health benefits of starting treatment earlier [10]. However, WHO recommended that nutritional care and support with macro/ micronutrients must be started at the early stages of the infection in order to prevent weight loss and malnutrition $[11,12]$.

\section{Food security and HIV/AIDS: The Nigerian story}

Food insecurity is associated with decreased ART adherence, reduced baseline CD4 cell count, incomplete virologic suppression, and decreased survival. Integration of food security interventions into HIV/AIDS treatment programs is essential to curtail the HIV/ AIDS epidemic and improve health and quality of life among those infected. Hence, the selection of Amtewa meal constituents was based on $[13,14]$ :

a. Literature sources and sound scientific researches on Nigeria indigenous plants with known macro and micronutrient compositions.

b. Plant based food sources in Nigeria that are readily available and affordable to ensure sustainability of the intervention program.

\section{Supplementation of Optimum Nutrient-Dense} Meal: the Amtewa Meal Case study

Nutrition interventions for PLHIV which are based on formulating nutrient-dense meals are innovative, culturally relevant, reliable and requiring low-tech approaches in order to assure compliance, sustainability and cost-effectiveness $[15,16]$. It is possible to improve the nutritive value of local foods through simple but scientific combinations of food ingredients in form of tailored functional recipes [TFR]. TFR is defined as food that is naturally occurring, accessible, affordable and perhaps consumed in unnatural concentrations as part of the usual diet and has demonstrated physiological and or biomedical benefits in reducing the risk of chronic disease beyond basic nutritional functions $[13,14]$.

Based on WHO recommendations on additional energy requirements for asymptomatic (10\%) and symptomatic $(20 \%$ - 30\%) PLWH and the EARs energy for male (2500 kcal/d) and female (2000 kcal/d), the average EARs was calculated as 2250 $\mathrm{kcal} / \mathrm{d}$. Since PLWH were advised to continue with their normal daily diet; an average of $10 \%-20 \%$ additional energy for both asymptomatic and symptomatic PLWH was calculated 3554.92 $\mathrm{kcal} / \mathrm{d}$ ). This additional energy (354.92 kcal/d) was formulated and provided in a $100 \mathrm{~g}$ sealed pack of Amtewa meal. The $100 \mathrm{~g}$ Amtewa meal containing $50 \mathrm{~g}$ soya beans, $15 \mathrm{~g}$ moringa leaves, $15 \mathrm{~g}$ of carrot roots and $20 \mathrm{~g}$ of millet which was dispensed to PLWH for daily consumption (up to 6 month) as illustrated in $[13,15]$.

Food security with Amtewa meal nutrition intervention has proven to be valid strategy to build the capacity of households and communities affected by HIV and AIDS to cope with the disease. Many other services and intervention programs exist, which are relevant but not primarily designed to address nutrient deficiencies HIV/AIDS in resource limited settings but with natural food sources available in the community such as in Nigeria.

\section{Food and Drug Interactions and Tolerability of HAART regimens}

Food and drug interactions are an important issue for effectiveness and tolerability of HAART regimens. Thus, in the Amtewa meal nutrition intervention, HAART regimens with documented evidence of interaction with food were monitored and the study participants on such regimen were counselled to use their medication 2-3hrs before or after the intervention meal. The presence of Amtewa meal in the gastrointestinal tract can influence the absorption of several HIV medications such as didanosine, indinavir, saquinavir, and nelfinavir. Drug-food interactions can influence serum drug concentrations, thus increasing the likelihood of side effects of the ART when serum concentrations are high and increasing the risk for viral resistance and loss of durable viral suppression when serum concentrations are low.

The Nigerian Guidelines for HIV and AIDS treatment and Care in Adolescents and Adults, the Federal Ministry of Health, Nigeria recommended strategies for improving and monitoring nutritional status. These recommendations include:

a. Close weight monitoring

b. Nutrition education and Counselling

c. Prompt treatment of opportunistic infections (mouth disorders, diarrhea)

d. Nutritional Support (macro and micronutrient)

e. Economic empowerment 
Although the guidelines recommended nutritional support with macro and micronutrients but no evidence of any intervention meal in all the treatment centres in Nigeria to ensure that the nutrient requirements for PLWH as recommended by WHO is achieved. Thus, Amtewa meal nutrition intervention when embedded into the guidelines on nutritional care and support for PLHIV in Nigeria suppressed the progression of HIV and provided indirect improvement of CD4 count. The results support the use of Amtewa meal as a TFR that is simple, inexpensive, and safe adjunct therapy in HIV disease management in resource limited settings.

\section{Conclusion}

There is still urgent need for social welfare and agriculture policies which will concentrate on food security and affordability especially in resource limited settings, where the malnutrition is pandemic problem contributing to the intensification of HIV symptoms. This review underpins nutritional support as an integral part of comprehensive response to HIV. It must, however, be emphasized that nutritional support cannot serve as substitute for antiretroviral therapy in resource limited settings.

Further research is needed to identify simpler effective interventions. Crosswise, nutritional assessment methodologies are needed that can be effectively utilised and adapted to the various programme and sustained service delivery models found in resource-limited settings in order to tailor micronutrient interventions related to the prevention, care and treatment of HIV/ AIDS.

\section{References}

1. Pribram V (2011) Nutrition and HIV. $1^{\text {st }}$ Edn. Wiley-Blakwell, West Sussex, UK

2. Ivers LC, Cullen KA, Freedberg KA, Block S, Coates J, et al. (2009) HIV/ AIDS, undernutrition and food insecurity. Clin Infec Dis 49: 1096-1102.

3. Amlogu MA, Tewfik S, Wambebe C, Tewfik I (2019) Innovative Nutritional approach to attenuate the progression of HIV to AIDS among People Living with hiv (PLWH): A study based in Abuja, Nigeria Jiaats 5(1).
4. Bamighausen $\mathrm{T}$, Chaiyachati $\mathrm{K}$, Chimbindi N (2011) Interventions to increase antiretroviral adherence in sub-Saharan Africa: a systematic review of evaluation studies. Lancet infec Dis

5. Boon NA, Walker BR (2006) Davidson 's principle and practice of medicines. $20^{\text {th }}$ Edn, USA.

6. Kumar P, Clark M (2005) Clinical Medicine $6^{\text {th }}$ edn. London: Elsevier Saunders Ltd.

7. UNAIDS (2019) Global AIDS Update 2019.

8. UNAIDS (2018) Global AIDS Update 2018.

9. Granich RM, Gilks CF, Dye C, De Cock KM, Williams BG (2009) Universal voluntary HIV testing with immediate antiretroviral therapy as a strategy for elimination of HIV transmission: a mathematical model. Lancet 373(9657): 48-57.

10. WHO (2015) A case for investing in Public Health: The strengthening public health services and capacity-A key pillar of the European regional health framework Health 2020.

11. Piwoz EG, Preble EA (2000) HIV/AIDS and nutrition: A review of the literature and recommendations for nutritional care and support in subSaharan Africa. SARA Project, Academy for Educational Development/ USAID, Washington DC, USA

12. WHO (2009) Nutritional care and support for people living with HIV/ AIDS: A training course. Geneva, World Health Organization.

13. Amlogu AM, Godden K, Tewfik S, Wambebe C, Tewfik I (2012) Tailored Food Recipe - TFR: Employing the European perspective on functional food science (FUFOSE) to promote effective dietary intervention in Africa. International Journal of Food, Nutrition \& Public Health 5 (1/2/3): 1-10.

14. Amlogu AM, Godden K, Tewfik S, Wambebe C, Tewfik I (2013) Public Health Nutrition Intervention Programme to Attenuate the Progression of HIV to AIDS among People Living with HIV (PLWH) in Abuja, Nigeria: A Conceptual Framework. International Journal of Food, Nutrition \& Public Health 6(1): 83-98.

15. Amlogu AM (2015) Public Health Nutrition Intervention to delay the progression of HIV to AIDS among People Living with HIV virus in Nigeria: A PhD thesis awarded by the University of Westminster.

16. Amlogu AM, Tewfik S, Wambebe C, Tewfik I (2016) A Comparative study: Long- and Short-term effect of a nutrition sensitive approach to delay the progression of HIV to AIDS among people living with HIV (PLWH) in Nigeria. Functional Food in Health and Disease 6(2): 79-90. 